\begin{tabular}{|c|c|}
\hline 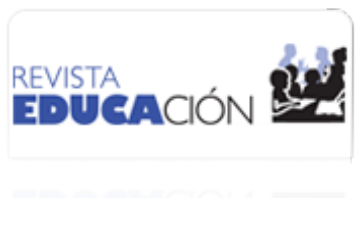 & $\begin{array}{l}\text { Revista Educación } \\
\text { ISSN: 0379-7082 } \\
\text { ISSN: } 2215-2644 \\
\text { revedu@gmail.com } \\
\text { Universidad de Costa Rica } \\
\text { Costa Rica }\end{array}$ \\
\hline
\end{tabular}

\title{
La educación universitaria en prisión: logros, dificultades y necesidades
}

\author{
Murillo Leiva, William \\ La educación universitaria en prisión: logros, dificultades y necesidades \\ Revista Educación, vol. 43, núm. 2, 2019 \\ Universidad de Costa Rica, Costa Rica \\ Disponible en: http://www.redalyc.org/articulo.oa?id=44058158026 \\ DOI: https://doi.org/10.15517/revedu.v43i2.31967
}

Esta obra está bajo una Licencia Creative Commons Atribución-NoComercial-SinDerivar 3.0 Internacional. 


\title{
La educación universitaria en prisión: logros, dificultades y necesidades
}

\author{
Prison College Education: Achievements, Difficulties and Needs
}

William Murillo Leiva

UNED, Costa Rica

wilmurilloleiva@gmail.com

(iD http://orcid.org/0000-0002-0951-0555
DOI: https://doi.org/10.15517/revedu.v43i2.31967

Redalyc: http://www.redalyc.org/articulo.oa?id=44058158026

Recepción: 11 Enero 2018

Aprobación: 02 Junio 2019

\section{Resumen:}

Este artículo es resultado de un proceso de investigación y exploración del proceso educativo de estudiantes universitarios en prisión. El objetivo de este artículo es presentar algunos de los principales logros y/o aciertos, dificultades y necesidades de la educación universitaria en prisión de acuerdo con la perspectiva de un grupo de estudiantes privados de libertad en diferentes centros de atención institucional (cárceles) del país. Se ha ejecutado de acuerdo con un diseño complementario de investigación, incluyendo una etapa cuantitativa y una cualitativa de exploración y construcción colectiva de saberes; la primera, determinada por un cuestionario como principal herramienta de recolección de información y aplicado en una muestra estadísticamente representativa de todo el estudiantado matriculado en la universidad y en las cárceles del complejo Reforma al momento de la aplicación de cuestionarios. La etapa cualitativa se ejecuta mediante un taller grupal con metodología de grupo operativo. Los resultados dan cuenta de una serie de logros de la educación universitaria en prisión y resaltan el impacto positivo de esta en el alumnado. Además, da cuenta de una serie de necesidades y dificultades propias del proceso educativo en dicho contexto. Se concluye, a partir del análisis de los resultados de esta investigación, a la educación en el contexto penitenciario como factor protector en torno a la cotidianidad de la institución total.

Palabras Clave: Educación, Educación superior, Prisión, Derechos Humanos.

\section{Abstract:}

This research article examines college education for students in prison, its achievements and/successes, difficulties and needs based on the perspective of a group of students incarcerated in various Costa Rican prisons. This study is based on a complementary research design and includes a quantitative and qualitative exploratory phase as well as collective knowledge construction. This was determined using a survey answered by a representative sample of the entire student body enrolled in the college program for La Reforma prison complex. The qualitative phase was conducted through an operational group workshop. The results show the great success of prison college programs and highlight the positive impact on the students. It also reveals existing needs and gaps inherent to this educational context. We can conclude, after analyzing the results, that college prison education is a protective factor for its students, helping to deter idleness as well as dangerous contexts associated with everyday prison life.

KEYWORDs: Education, College Education, Prison, Human Rights.

\section{INTRODUCCIÓN}

Este artículo es producto y presenta algunos de los principales resultados de un proceso de investigación del proceso educativo de estudiantado universitario en prisión. Su principal objetivo es presentar logros, dificultades y necesidades principales de la educación universitaria en prisión y de acuerdo con la perspectiva del alumnado de la muestra de investigación; buscando así una mejor comprensión de la experiencia universitaria en prisión. Esto es relevante para el campo o área científica y profesional de la educación porque permite profundizar y reflexionar al respecto de los procesos educativos y sus necesidades y dificultades, así como algunos de los aciertos y logros de la educación superior en el complejo penitenciario La Reforma. 
Lo anterior, con el propósito de conocer con detalle cómo es que se desarrolla en la actualidad la oferta educativa de la UNED en los centros penales objeto de este estudio y para, finalmente, promover cambios, mejoras y el reconocimiento de aquellas cuestiones que deben atenderse con urgencia.

Los resultados presentados se organizan de acuerdo con categorías específicas de aspectos significativos. En este sentido, este documento da cuenta de: a) aspectos sociodemográficos de la muestra participante b) logros y/o aciertos, b) dificultades y, c) necesidades clave de la educación universitaria en prisión. La información presentada se fundamenta, principalmente, en los resultados obtenidos de la etapa cuantitativa de investigación.

Así, la investigación explora, de acuerdo con la perspectiva estudiantil, el impacto de cursar estudios universitarios en distintos centros penitenciarios, las implicaciones de estos procesos educativos en su cotidianidad y enfatiza en detalles y aspectos importantes de la experiencia del alumnado de la Universidad Estatal a Distancia de Costa Rica (en adelante UNED).

Específicamente, se defiende a la educación universitaria o superior como derecho y se reclama o puntualiza la obligación del Estado costarricense de garantizar a las personas privadas de libertad el mismo derecho con que cuenta el resto de la ciudadanía, en este caso, el acceso a una educación superior de calidad y de acuerdo con las condiciones de las personas internas.

Además, analiza a la educación en el contexto de la prisión como una herramienta para el desarrollo personal que facilita y potencia la inclusión social y laboral tanto dentro como fuera del centro penitenciario, promoviendo así una sociedad más equitativa y más justa a través de un adecuado proceso de inserción social de las personas privadas de libertad.

La oportunidad universitaria brindada por la UNED Costa Rica en prisiones “[...] es notablemente valiosa y refleja una voluntad política y un criterio profesional y académico con una perspectiva coherente con los Derechos Humanos [...]" (Murillo, 2017, p. 61). Esta universidad ha asumido de manera sobresaliente y coherente con una perspectiva de Derechos Humanos la oferta universitaria en todas las cárceles del país; demostrando interés por una población en especial marginada y estigmatizada. Este esfuerzo es valioso y potencia el desarrollo de seres humanos con ganas de aprender y estudiar; posibilitando así la mejora en su cotidianidad, la de sus familias y la de la sociedad en general. Ahora bien, la oferta universitaria debe innovar, ampliar y promover la mejora integral de sus servicios, conocer la dirección que deben tener estas mejoras es parte del interés y la justificación que dio pie a la investigación base de esta publicación.

Con respecto a lo anterior vale destacar que dicho trabajo se elabora a través del Programa de atención de estudiantes privados de libertad. La oferta se brinda a cualquier persona que cumpla los requisitos académicos y el costo económico, y desee cursar estudios superiores en la cárcel.

Normalmente, la UNED de Costa Rica, brinda gran parte de su oferta educativa, -que es a distancia- a través de plataformas digitales y en línea. En algunos casos, los cursos no se desarrollan de manera virtual y se imparten mediante tutorías presenciales, videoconferencias o recursos semejantes. Además, muchas de las asignaturas, de las múltiples carreras de la universidad, complementan el acompañamiento académico brindado de manera virtual a través de tutorías presenciales.

Es importante destacar que la población privada de libertad en Costa Rica, incluyendo estudiantes universitarios, tienen prohibición total de acceso a internet y, por ende, no pueden acceder a las plataformas virtuales de la Universidad en sus centros penales.

Así las cosas, el Programa de atención de estudiantes privados de libertad en conjunto con actores claves, es decir: funcionarios y docentes de cada carrera respectiva, deben proveer al estudiantado universitario en prisiones costarricenses de materiales didácticos y bibliográficos necesarios para su óptimo desempeño. Además, se le debe facilitar a cada estudiante las pruebas y todo tipo de evaluación académica que implique la asignatura o asignaturas cursadas, adaptadas desde de la virtualidad cuando corresponde y para su contexto.

Finalmente, en ocasiones pueden recibir tutorías, pero dependen de la cantidad de estudiantes matriculados y en muchas ocasiones, de la voluntad de los encargados académicos. 


\section{EsTADO DE LA CUESTIÓN}

\section{Necesidades}

La investigación con respecto a la educación en prisión presenta algunos antecedentes valiosos para el trabajo desarrollado en este documento y recalcan la complejidad y especificidad de dicha oferta (Scarfó, Cuellar y Mendoza, 2016).

Sobresale que la educación en centros penitenciarios encuentra multiplicidad de dificultades, por ejemplo: exiguo material de estudio adaptado al alumnado adulto y la insuficiencia y caducidad de los materiales disponibles, carencia de un diseño curricular adecuado, escasa formación pedagógico-didáctica específica de los docentes para actuar en el contexto de centros penitenciarios, entre otros (Blazich, De Milén y Viedma, 2007).

Así (Viedma, 2003) ha asegurado que la imposibilidad de utilización de medios y recursos habituales en la población de estudiantes universitarios en prisión con respecto a población que no está en prisión se constituye como una clara desventaja para los primeros y se señala que es necesario ofrecer medios y materiales más adecuados. Por ejemplo, el autor destaca la insistencia de petición de mayor ayuda de tutores.

Entonces, en la educación universitaria en prisión, de acuerdo con Viedma (2013) "Los medios básicos como la bibliografía, el apoyo de tutores o la comunicación con los equipos docentes son exiguos" (p. 145). Y, se destaca, que la resistencia de estas instituciones penitenciarias a incorporar TIC en los procesos educativos resalta el lugar secundario que se le da a la educación por debajo de la seguridad penitenciaria (Viedma, 2013).

Aunado a lo anterior, la educación universitaria en prisión, distingue una práctica educativa que mayoritariamente es adversa a la educación superior y a las complejidades y dificultades que implica su desarrollo en los centros penales (Gutiérrez, Callejo, y Viedma, 2010). "Se trata de prácticas conservadoras caracterizadas por situar la redención del preso por encima de su formación y rehabilitación” (p. 465).

En esta línea, pero refiriéndose a la educación en prisiones argentinas, López (2009) destaca que la educación en cárceles de Buenos Aires no es ordenada, articulada ni integral. Además, en la misma línea de lo ya mencionado, destacan limitaciones en disponibilidad de material educativo actualizado, problemas en acceso a información sobre la oferta educativa y desarrollo de educación sin el adecuado y óptimo acceso a TIC en las cárceles argentinas (Scarfó y Zapata, 2013).

También se visualizan muchas de estas deficiencias en análisis brasileños con respecto a la educación en prisiones de ese país; así, como se ha mencionado, la educación en prisión se caracteriza por falta de material didáctico adecuado, escases de recursos pedagógicos y limitaciones infraestructurales (Bandeira, et ál, 2008).

A nivel nacional, y específicamente hablando de educación superior, se caracteriza que una educación universitaria en prisión presenta dificultades para potenciar la atención y la comprensión, que los recursos didácticos no son los adecuados y que no se reciben tutorías para aclarar dudas y comprensión de materia (Castrillo, 2012).

Además, con una falta de infraestructura adecuada para atender los estudios, el acceso a los medios virtuales $\mathrm{y}$, en general, a materiales de apoyo que se ofrecen en los cursos, son dificultados por las mismas condiciones de control del sistema carcelario que imposibilitan ejecutar una mediación pedagógica más eficaz (Ulate y Vargas, 2012).

También, se destaca que el acompañamiento al estudiante y el entendimiento -por parte de la institución educativa y el profesorado- de que la población tiene especificidades y necesidades propias de su contexto es trascendental para el éxito académico (Rivera, 2016).

Todo lo anterior está muy relacionado y es congruente con los resultados de la investigación aquí presentada. Se destacan dificultades y necesidades de los procesos educativos en prisión que en este trabajo son confirmadas y visualizadas de acuerdo a la perspectiva de las y los estudiantes. 
El estado de la cuestión ratifica que la educación dentro de los centros penitenciarios es compleja y que se debe profundizar en la misma para proponer y desarrollar cambios y mejoras que faciliten su desarrollo; no solo pensando en la reincorporación del privado de libertad en la sociedad sino también en la garantía y defensa de un Derecho Humano.

\section{MARCo TEÓRICo}

Esta investigación parte de la teoría de los Derechos Humanos. Los Derechos Humanos son el "conjunto de procesos sociales, económicos, normativos, políticos y culturales que abren y consolidan -desde el reconocimiento, la transferencia de poder y la mediación jurídica- espacios de lucha por la particular concepción de la dignidad humana" (Herrera, s.f., citado en Camacho, 2009, p. 15). Y, sobre todo, se destaca que "más que una idea universal es un proceso de lucha por la dignidad general y particular, de personas y grupos con una trama de relaciones específicas según el momento y el lugar" (Camacho, 2016, párr. 6).

Bajo este fundamento se aproxima este trabajo a la persona privada de libertad, como ser humano, por ende, digno y con derecho a la educación y, a la educación en este contexto como un factor esencial y como espacio de lucha para el reconocimiento de dicha dignidad.

El derecho a la educación, además, es un derecho complejo en el que, tal y como lo destaca De Castro (2004), la acción de los poderes públicos en relación con este derecho no puede ni debe limitarse a la prestación del servicio sino que, el estudiantado deben disponer de posibilidades reales para acceder a la educación en las condiciones idóneas para obtener el provecho respectivo.

Así, es la educación en el contexto penitenciario un derecho trascendental para el desarrollo y la condición humana. Además, un derecho que debe establecerse de acuerdo con una serie de condiciones (Scarfó, 2011). En este sentido, las reglas mínimas de las Naciones Unidas para el tratamiento de los reclusos (Organización Mundial de las Naciones Unidas, 2016), destacan en la regla 4 que "Los objetivos de las penas y medidas privativas de libertad son principalmente proteger a la sociedad contra el delito y reducir la reincidencia” (p. 3) y que, "Para lograr ese propósito, las administraciones penitenciarias y otras autoridades competentes deberán ofrecer educación, formación profesional y trabajo, así como otras formas de asistencia apropiadas y disponibles" (p.4).

Entonces, la educación es un derecho humano clave para la consecución del desarrollo personal, humano y social. "El derecho a la educación -que no es sino el derecho a aprender- está en el centro de la vida de la persona y de la sociedad. Es lo que permite el desarrollo armónico de las facultades del ser humano" (CPEUM 2014, citado en Instituto Nacional para la Evaluación de la Educación [INEE], 2014, p. 10).

Además, se entiende a la privación de libertad como una acción que, si bien se establece ante un hecho tipificado como delito no debe privar más derechos que el de la libertad de tránsito. Así, menciona Scarfó (2011) “(...) el encarcelamiento, aunque se considere un castigo justificado, no debe llevar consigo una privación adicional de los otros derechos, ya que el único derecho que se priva, al estar detenido, es la libertad ambulatoria" (p. 25).

Aunado a lo anterior, como menciona Arias (1996/1997). "el ingreso a la prisión de un sujeto significa ni más ni menos que la restricción de su libertad ambulatoria y de algunos otros derechos conexos, pero conserva la mayoría de los derechos del ciudadano común" (p. 44).

Así, este trabajo se aproxima al contexto penitenciario como un espacio de administración de la pena que debe cumplir con una serie de condiciones y de derechos inherentes a la persona humana y que no puede superar las funciones para las cuales se establece. Si bien está claro que la privación de la libertad significa un contexto de multiplicidad de limitaciones y de gran impacto en la vida y desarrollo de las personas internas, la educación, en este contexto, debe superar a las limitaciones del centro para establecerse como corresponde y en su calidad de derecho (Goffman, 2001). 


\section{Metodología}

La metodología de la investigación se comprende como un diseño complementario de aproximación al objeto de estudio; es decir, desarrolla una etapa cuantitativa y una cualitativa de recolección y construcción de datos.

El proceso metodológico, en el campo, implicó acceder a todos los ámbitos o sectores de las prisiones del complejo Reforma en dónde existieran y estuvieran disponibles estudiantes matriculados en la oferta de educación superior de la UNED Costa Rica. Con la gran mayoría de estos se llevó a cabo la etapa cuantitativa de la investigación. La etapa cualitativa reunió a un grupo menor de estudiantes en un espacio especial dentro del centro penal y para profundizar en sus experiencias como estudiantes universitarios en prisión.

La etapa cuantitativa se desarrolla mediante la aplicación de un cuestionario, diseñado con base en investigaciones previas (Rivera, 2016; Blazich, et ál, 2007; Castrillo, 2012; entre otros), a una muestra estadísticamente representativa de toda la población de estudiantes privados de libertad matriculados en los centros de atención institucional (centros penitenciarios) que forman parte del complejo Reforma. Vale aclarar que el programa de educación superior para estudiantes privados de libertad de la UNED Costa Rica se desarrolla en varios centros penitenciarios y de diversas provincias del país. Así, esta investigación se desarrolla específicamente en el complejo Reforma; que reúne a los principales centros penitenciarios masculinos de la República de Costa Rica.

Específicamente: un 38,1\% está privado de libertad en Centro de Atención Integral Jorge Arturo Montero Castro, un 33,3\% en el CAI Dr. Gerardo Rodríguez, un 22,2\% en el CAI Luis Paulino Mora, un 4,8\% en APAC y un 1,6 en CAI Adulto Mayor. Ver Figura 1.

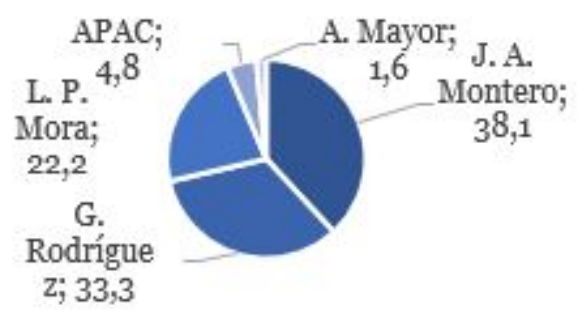

FIGURA 1

\section{Ubicación de Participantes en Centros de Atención Institucional \\ Fuente: Elaboración propia}

Con respecto a la etapa cuantitativa, participó una muestra estadísticamente representativa, $(\mathrm{n}=63)$ de la población total de 68 estudiantes universitarios de los centros penitenciarios del Complejo Reforma. Determinada así por el programa STATS 2.0 con un porcentaje de error de $5 \%$ y un nivel de confianza de 99\%. El análisis de la información cuantitativa se efectuó mediante el programa estadístico SPSS V18.

La etapa cualitativa se ejecutó mediante un taller grupal con metodología de grupo operativo que profundiza en la experiencia subjetiva del proceso estudiantil de los participantes. Este grupo se organizó con 10 participantes que fueron parte de la primera etapa de investigación y que mostraron interés y voluntariedad en participar en esta segunda etapa.

Los datos obtenidos y construidos en conjunto con los participantes se presentan y analizan de acuerdo a estadísticas descriptivas y análisis del discurso (en el caso de los datos de la etapa cualitativa o las preguntas abiertas del cuestionario de investigación), sistematizados por categorías de análisis. 


\section{ANÁLISIS Y DISCUSIÓN DE RESULTADOS}

\section{a) Aspectos sociodemográficos}

TABLA 1

Algunos aspectos sociodemográficos

$\begin{array}{llll} & \text { Costarricense } & \text { Otra } & \\ \text { Nacionalidad } & 92,1 \% & 7,9 \% & \\ & \text { Soltero } & \text { Casado } & \text { Otro } \\ \text { Estado civil } & 52,4 \% & 20,6 \% & 27 \% \\ & \text { Primaria } & \text { Reincidente } & \\ \text { Condición } & 84,1 \% & 15,9 \% & \\ \text { penitenciaria } & & & \end{array}$

Fuente: Elaboración propia

Con respecto a algunos resultados sociodemográficos de la investigación, en la Tabla 1 , se determina, en cuanto a la nacionalidad que, un $92,1 \%$ de la muestra indica ser costarricense (esto es 58 personas), mientras que el $7.9 \%$ se distribuye en otras nacionalidades. Al cuestionárseles por su estado civil, un 52, 4\% comparte que es soltero y un 20,6\% casado. Con respecto a la condición penitenciaria, la población universitaria, en su gran mayoría, cumple condena por primera vez $(84,1 \%)$. Siendo significativo este dato en tanto refleja que hay un interés mayoritario hacia la educación universitaria en privados de libertad por primera vez recluidos. Además, en cuanto a la paternidad, en términos de mayorías, la mayor parte de la muestra participante, un $34,9 \%$, indicó no tener hijos; un 15,9\% da cuenta de 1 hijo y un 14,3\% responde que posee 2 .

\section{b) Logros y aciertos}

\section{Acceso a la educación}

En primera instancia, es importante destacar, como logro, que la población privada de libertad, cumpliendo con los requisitos académicos, es decir, habiendo aprobado los grados previos, puede matricularse en la educación superior sin mayor complejidad o dificultad; además, puede optar por una beca que cubra con sus gastos de los créditos o asignaturas; garantizándose así la posibilidad de ser estudiante de educación superior en prisión. Así, la oferta es estable y permanente; además, de un acceso considerablemente adecuado; esto guarda relación con el valor y la importancia que han destacado investigaciones previas en torno a considerar como un éxito que la oferta universitaria en prisión sea estable y que resulte incuestionable en la actualidad (Viedma, 2003).

Específicamente relacionado con la obtención de beca; un 95,2\% menciona que posee beca y por su parte, solo un 3,2\% dice no disfrutar de dicho beneficio. Reflejando este dato excelentes números con respecto a la cobertura económica que brinda la universidad a sus estudiantes. Además, con respecto a la posibilidad de obtener esta beca, ante la pregunta ¿Qué tan fácil resulta obtener beca?, un 27\% considera que es fácil, un $34,9 \%$ que es regular y un 33,3\% de la muestra indica que es difícil o muy difícil. Ahora bien, este porcentaje $(33,3 \%)$, es un dato que puede ser significativo a la hora de analizar la experiencia de las y los estudiantes puesto que, evidentemente, la beca facilita el pago de los créditos matriculados; ¿se pueden hacer cambios o mejoras para facilitar el acceso a la beca?, ¿es posible alguna modificación en términos de reglamento? El 
planteamiento y la búsqueda de respuestas ante estas preguntas permitirían reflexiones y construcción de mejoras oportunas.

\section{Acceso a información sobre oferta universitaria}

Manteniendo la presentación y discusión de resultados al respecto de logros y/o aciertos de la educación universitaria en prisión, resulta valioso reflexionar acerca del acceso a información con respecto a los estudios y visualizar algunos de los resultados relacionados con esto.

La información es vital para la construcción del conocimiento y de decisiones informadas, además, es un Derecho Humano enmarcado en el derecho a la expresión que, de acuerdo con la Convención Americana sobre Derechos Humanos, en su artículo número 13 (Organización las Naciones Unidas, 2015).

Los resultados demuestran que un 76,2\% menciona haber recibido información -antes de ingresar a la universidad-acerca de las carreras que ofrece la universidad, cursos y carreras restringidas, entre otro tipo de información; porcentaje amplio y mayoritario.

Da cuenta lo anterior de que, en relación con la experiencia universitaria, previo a la matrícula, la mayoría de las y los estudiantes cuentan con algún tipo de información, constituyéndose esto como un logro, aunque está claro que existe una gran cantidad de personas que no recibe apoyo informativo alguno $(23,8 \%)$.

Con respecto a qué persona o entidad le brindó la información mencionada, destaca el papel del área educativa del centro penal $(31,7 \%)$ y, de los compañeros internos $(36,5 \%)$ como los principales emisores de información con respecto al contexto universitario. Dato significativo y que rescata la importancia de las personas presentes en el contexto carcelario y en especial de los compañeros a la hora de decidir iniciar la experiencia universitaria.

Vale destacar que, usualmente, las y los estudiantes reconocen al encargado o designado del Centro Universitario como funcionario del área educativa y esto visualiza la importancia del funcionario de la UNED en este caso, que está presente en la prisión y con respecto al proceso académico del estudiantado universitario.

En el contexto penitenciario el acceso a información resulta limitado y la oferta universitaria debe atender esta complejidad y promover el acceso igualitario a la información. Las dificultades en torno al acceso a internet y a información académica en prisión atentan contra el disfrute del derecho a la información y al de la educación. Aspecto que, como se ha destacado, es muy importante, la información es trascendental en cualquier proceso de decisión. ¿Cómo se toma la decisión sin ninguna información al respecto? Probablemente se toma motivado o influenciado por información deficiente y escasa. Esto plantea trabajos comprometidos y activos para con la administración universitaria y penitenciaria dirigidos a facilitar el acceso a la información a las personas privadas de libertad.

El acceso a información con respecto a la oferta educativa por parte de estudiantes en prisión también ha sido destacado por trabajos previos que hablan de la necesidad y de la importancia de procesos de comunicación y de trasmisión de información adecuados y oportunos (Scarfó y Zapata, 2013). En el caso de esta investigación se visualizan resultados positivos en torno a la disposición de la información con respecto a la oferta educativa, pero sin duda también se reflejan inminentes necesidades de mejora.

\section{Impacto psicosocial}

Es significativo resaltar de qué manera estudiar mientras se cumple condena en prisión ha influido en la vida de los participantes. Aspectos que permiten considerar a la experiencia universitaria en prisión, a la educación en esta como un factor protector o, como un Lugar seguro; como una oportunidad que genera una sensación de mayor seguridad, bienestar y satisfacción, es quizás uno de los aspectos más relevantes que este trabajo, conforme a sus resultados, plantea. Se considera lugar seguro, por un lado, porque el aula dispuesta para las 
y los estudiantes se encuentra en un sector diferente al de los dormitorios y esto expone mucho menos a las y los estudiantes a los conflictos o problemáticas que en los dormitorios se generen y, por otro lado, porque al ocupar el tiempo en el estudio los hace involucrarse menos en situaciones de riesgo, consumo de drogas, violencia o peligro. Además, se entiende que la educación y el desarrollo personal potencia una convivencia más consciente, humana y pacífica consigo mismo y el entorno.

Así, sobresale que, ante la pregunta: ¿De qué manera ha influido estudiar en prisión en su vida? Un 69,8\% menciona que de manera muy positiva y un $27 \%$ de manera positiva. Por su parte, un $1,6 \%$ determina ha impactado de manera negativa y también un 1,6\% dice que no ha tenido ningún impacto. Entonces, un 96,85 de las y los estudiantes destaca el impacto positivo de los procesos educativos universitarios en su vida de internamiento.

En la misma línea de los resultados anteriores, es muy significativo destacar que, ante la pregunta, ¿en qué grado considera que estudiar durante su tiempo de condena ha influido positivamente sobre su vida en prisión? un $54 \%$ y un $9.5 \%$ de la población encuestada ha mencionado Mucho y Bastante, respectivamente, cuando se le ha presentado la opción Me ha impedido dedicarme a consumir drogas. Es decir, un 63,5\% de la muestra considera que la educación universitaria en prisión ha influido en gran manera para que no consuman drogas. Se tiene claro que el consumo de drogas es un fenómeno complejo y multicausal, pero se logra visualizar un impacto positivo de la educación en el consumo de drogas dentro del centro penal de acuerdo con la perspectiva de los participantes, consumo que es evidente en centros penales y que tiene muchas implicaciones perjudiciales con respecto a la dinámica del encierro.

En una línea semejante, ante la frase Me ha servido para alejarme de los peligros de la vida en prisión, se ha contestado con Mucho en un 57.1\% y con Bastante en un 25,4\% de los sujetos participantes (ver Figura 2). Lo anterior corresponde a un $82,5 \%$ de la muestra y que refuerza el papel significativo de la educación universitaria en el contexto penitenciario para intervenir la cotidianidad y, específicamente, para resguardar con respecto a los peligros en prisión. Sobresale así a la educación universitaria en prisión como un factor que protege al estudiante, como un lugar seguro.

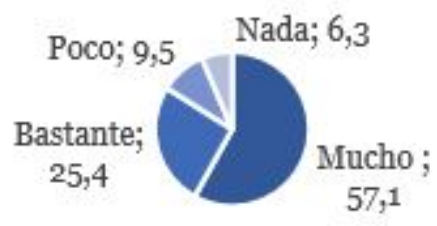

\section{FIGURA 2 \\ Estudio como protector con respecto a peligros en prisión \\ Fuente: Elaboración propia}

Entonces, ha podido visualizarse una educación la cual interviene la dinámica del consumo de drogas en el centro penitenciario y que, en consideración de la mayoría de la muestra, les aleja de los peligros de la vida en prisión o que, de alguna manera, mejora sus condiciones de vida. El énfasis de las y los estudiantes al solicitar que se amplíen los horarios de permanencia en el aula o la creación de aulas específicas de la universidad, cuando el centro penitenciario no cuenta con estas, está relacionado con este aspecto, no solo habla del interés por el estudio si no también, por estar, la mayor parte del día, en un espacio que les da una sensación de mayor seguridad. Así, la educación es una herramienta que da mayor salud mental y sensación de bienestar mediante la interpelación a la cotidianidad penitenciaria que impacta con monotonía, pérdida de la autonomía y ociosidad, entre muchas otras cuestiones (Goffman, 2001).

Es el aula, un espacio diferenciado, de protección, que en cierta manera abstrae de la cárcel y la dinámica penitenciaria. Resulta poderosa esta función de la educación en el contexto penitenciario, trasciende la posible reintegración en la dinámica social y laboral, protege a las personas mientras se encuentran 
cumpliendo condena. Esto es una consideración que emerge de los resultados obtenidos y que no se ha visualizado ni categorizado de esta manera ni con este nivel de especificidad en la investigación previa.

Además, podría considerarse que no solo impacta en términos de una sensación de mayor protección si no que, inclusive, de libertad o al menos, de una alternativa o interpelación al castigo. Podría decirse que la intención de permanecer más tiempo en el aula no solo hace sentirse al estudiante más seguro o protegido, sino también, más libre. Esta libertad depende, eso sí, de modelos educativos que promuevan y posibiliten que el estudiantado alcance autonomía y que tenga un lugar creador y liberador en su proceso educativo (Murillo, 2017).

También, es un acierto o logro de la educación en prisión a la posibilidad que brinda a los internos de ocupar el tiempo de mejor manera. Así, la opción Me ha servido para ocupar el tiempo ha arrojado que, de acuerdo con la Figura 3, un 95,3\% de la muestra valora a la educación como una opción para interpelar la ociosidad absurda del régimen penitenciario; también destacando y permitiendo visualizar la educación teniendo un impacto claro y positivo en la experiencia carcelaria. Entiendo la ociosidad en las prisiones como un aspecto generador de daño psicológico, donde la educación suma como elemento protector en contra del deterioro mental de las y los estudiantes (Goffman, 2001).

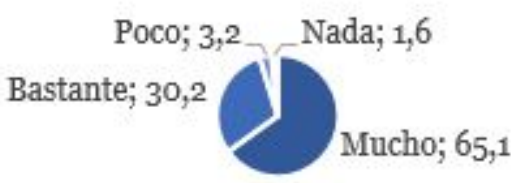

\section{FIGURA 3 \\ Estudio como herramienta para ocupar el tiempo disponible en prisión. \\ Fuente: Elaboración propia}

Además, vale resaltar la opción Me ha servido para mejorar mis condiciones de vida que ha presentado resultados de 55,6\% contestado con Mucho y 30,2\% con Bastante. Entonces, un 83,5\% de las y los estudiantes otorga a la educación universitaria en prisión un rol y, en cierta manera una competencia para mejorar las condiciones de vida.

También, ante la cuestión de Ha mejorado mis relaciones con mi familia un 46\% indica Mucho y un 33,3\% Bastante. Es decir, un 79,3\% destaca a la educación como factor potenciador de las relaciones familiares de las y los estudiantes. Demostrándose así otro de los alcances de la educación universitaria en prisión.

Además, al hablar de impacto psicológico y psicosocial de la educación en prisión. Se puede destacar como logro o alcance de la educación universitaria en prisión la posibilidad que brinda al interno de sentirse mejor consigo mismo o de sentirse a gusto puesto que favorece su desarrollo a través de esta oportunidad. Las y los estudiantes, en este sentido y al respecto de la consulta ¿Cuál cree que ha influido en mayor medida sobre su interés de volver a estudiar en prisión? Destacan, en un 47,6\%, porcentaje mayoritario de las respuestas, que su razón principal es la satisfacción personal.

En relación directa con lo anterior, un 44,4\% y un 12,7\% se ha manifestado De acuerdo y Muy de acuerdo respectivamente a la hora de considerar que Seguir estudios en la cárcel es una cuestión de satisfacción personal. Es decir, un $57,1 \%$ de la muestra entrevistada destaca a la satisfacción personal en relación directa con el estudio en prisión. Así, sobresale la educación universitaria en prisión como elemento satisfactorio. Como una decisión que se establece para estar mejor para sentirse bien, como ya se ha destacado. Esto coindice con lo mencionado por Blazich, et ál (2007), al decir que:

La posibilidad de estudiar en estos contextos, va más allá de una preparación del/la interno a la sociedad o un vínculo laboral futuro; tiene que ver con la recuperación de un derecho, el de la educación, y con la normalización de la vida cotidiana 
durante el cumplimiento de la condena; en este sentido su re construcción está relacionada con la satisfacción personal o las expectativas futuras (p.3)

También se destaca con un 30,2\% Deseo preparar mi reinserción laboral como razón principal en torno al interés de estudiar en prisión. Siendo, después de la satisfacción personal, la razón con más porcentaje de elección. Lo anterior impone la concepción de la educación en el contexto universitario como algo que trasciende a una mera obligación o a una opción para descontar años de condena. Estas razones se detallan en la Figura 4.

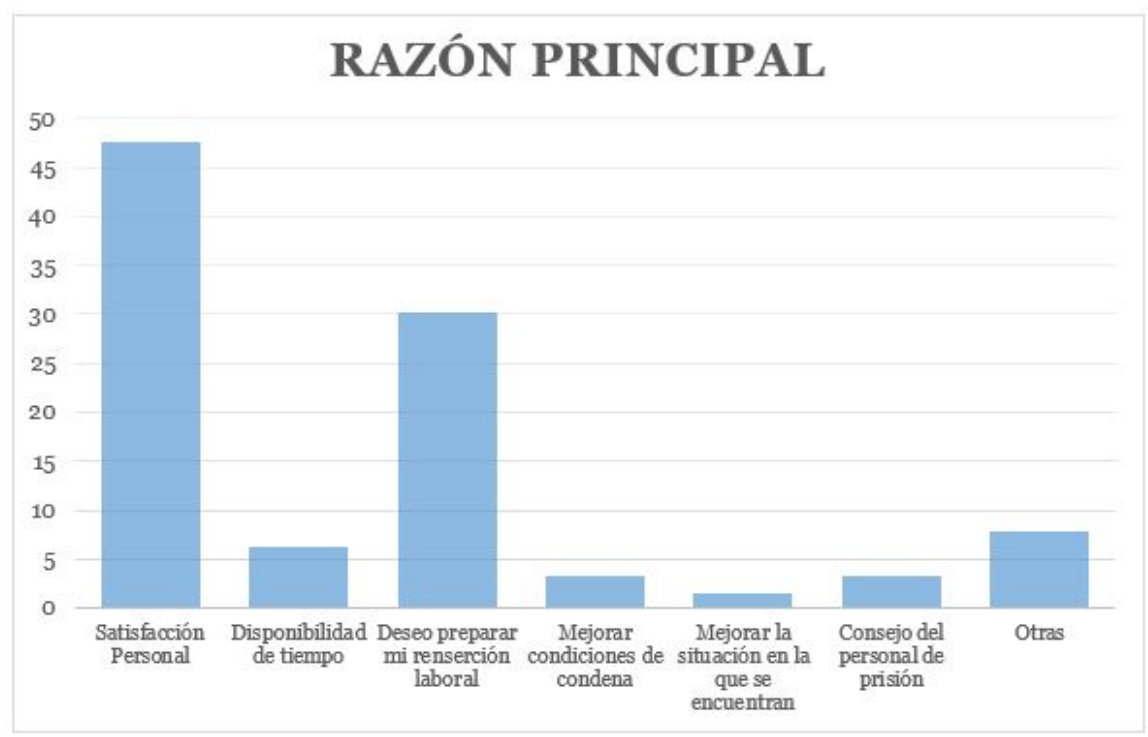

\section{FIGURA 4 \\ Razón principal para cursar estudios universitarios en prisión \\ Fuente: Elaboración propia}

\section{Permisos y tiempo suficiente para el estudio}

Con respecto al tiempo con el que cuentan para dedicarse a los estudios un $60,3 \%$ evaluó las condiciones como adecuadas y un $14,3 \%$ como muy adecuadas. Por su parte, solo un $14,3 \%$ y un $11,1 \%$ determinan que el tiempo para estudiar es bastante o muy inadecuado respectivamente. Además, ante la pregunta ¿Cuenta con permisos y un horario de estudio establecidos por el centro penal?, un $68,3 \%$ ha indicado que sí, mientras que un $30,2 \%$ que no y una persona no ha respondido la pregunta. Esto denota a una gran mayoría con permisos y horarios de estudio, pero al mismo tiempo permite visualizar un porcentaje elevado de estudiantes que no cuenta con dichas condiciones o posibilidades; otro de los aspectos por atender con rigurosidad y buscando la equidad y la igualdad en términos de las condiciones para la realización del derecho.

\section{c) Dificultades}

\section{Escaso apoyo académico}

Considerando el apoyo y el acompañamiento académico trascendental en los procesos educativos en los centros penitenciarios, en tanto son instituciones cerradas que rompen o dificultan los lazos de interacción, en este caso con la universidad, cuerpo docente y familiares, resulta significativo puntualizar, comprender 
y reflexionar al respecto de este apoyo y acompañamiento que los participantes de la muestra retratan. El apoyo de la pareja y/o familia la que es calificada, por un 76,2\% de la muestra, con valoración de 10 y que corresponde a la mayor calificación posible con respecto al apoyo brindado. Sin embargo, el segundo elemento con mayor calificación perfecta corresponde al apoyo que brindan los funcionarios de la universidad con un $27 \%$ de valoraciones de 10 .

Así, las instancias mejor calificadas como fuentes de apoyo, según se presenta en la Figura 5, son la pareja y/o la familia con una media de 8,19; y muy por debajo: los funcionarios de la universidad con una media de 5,59; el área educativa con una media de 4,95 y, la Defensoría de estudiantes de la UNED con una media de 4,44. El Programa de Atención a Estudiantes Privados de Libertad de la Universidad Estatal a Distancia, por su parte, obtuvo una media de calificación de 4,02 y los compañeros internos de 3,75.

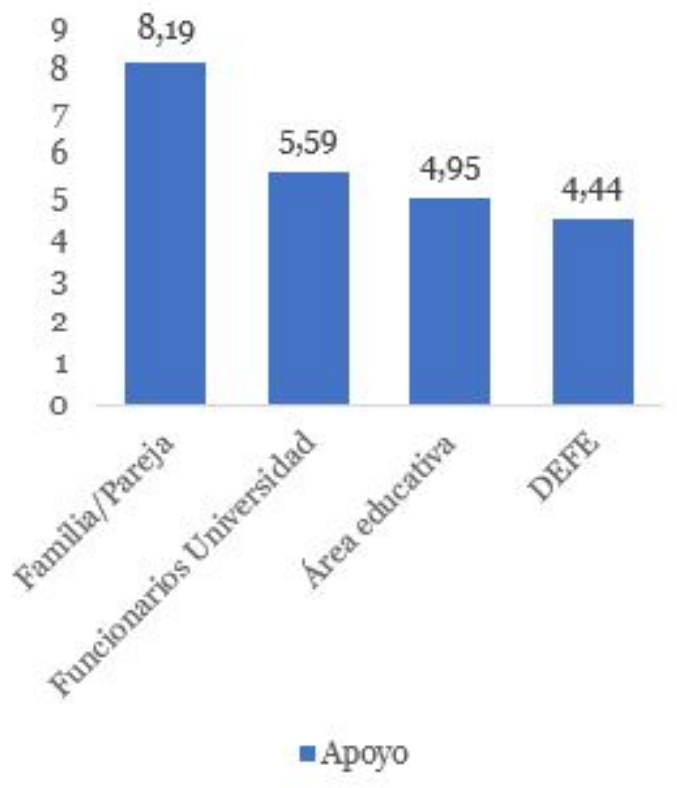

FIGURA 5

Fuentes de apoyo recibido por parte de las y los estudiantes

Fuente: Elaboración propia

Ahora bien, con respecto a la pregunta ¿Cómo califica el acompañamiento que recibe?, un 34,9\% lo considera regular, un $19 \%$ malo y muy malo un $14,3 \%$, esto es: $33,3 \%$ de la muestra otorga mala calificación al acompañamiento. Así como, un 17,5\% y un 14,3\% lo califica como bueno o muy bueno respectivamente. Así, un $68,2 \%$ de la muestra lo considera regular o deficiente. Resulta esto muy significativo y pone énfasis en que la experiencia universitaria está siendo marcada por un acompañamiento limitado y con grandes posibilidades de mejoría. Ante la consulta ¿considera que el acompañamiento que recibe es?, $44,4 \%$ indica que lo considera escaso y un 36, 5 lo valora como muy escaso; es decir, un $80.9 \%$ de la muestra visualiza e indica algún nivel de escases en su acompañamiento académico como estudiante universitario en prisión. Lo anterior se presenta en la Figura 6. 


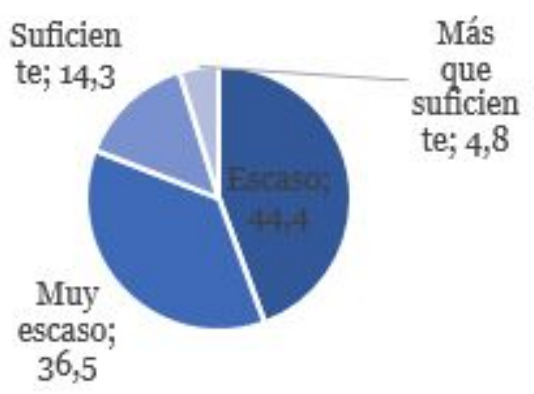

FIGURA 6

Consideración al respecto del acompañamiento vivenciado en la experiencia universitaria. Fuente: Elaboración propia

Queda clara la necesidad percibida por parte de la muestra con respecto al acompañamiento y la importancia de potenciarlo y promoverlo. El estudiantado refiere en reiteradas ocasiones la necesidad de un mayor acompañamiento e insta a la universidad a reforzarlo.

\section{Deficiencias en espacio físico}

Con respecto a los espacios para estudio un 30,2\% y un $27 \%$ los determinan como Muy inadecuados y Bastante inadecuados respectivamente, esto es un $57,2 \%$. Reflejando lo anterior que la mayoría de la muestra de estudiantes considera que las condiciones de los lugares de estudio son, en alguna medida, deficientes. Por ejemplo, las y los estudiantes mencionan "Cuando estoy en los módulos no hay espacios adecuados y en la noche no hay luz o iluminación" (Privado de libertad \# 5, comunicación persona, febrero, 2017) o "El tener que estudiar la mayor parte del día en uno de los peores lugares donde el ruido, los malos olores, los distractores y otros son el pan de cada día" (Privado de libertad \# 9, comunicación persona, febrero, 2017).

En relación con lo anterior y ante la pregunta ¿Su lugar de estudios cuenta con los requerimientos que usted necesita para estudiar? Un 66,7\% menciona que no y por su parte un 31,7\% indica que su lugar de estudios sí cuenta con los requerimientos necesarios para estudiar.

En esta misma línea, el espacio de biblioteca, por su parte, se destaca como Muy inadecuado en un 39,7\% de los casos y Bastante inadecuado en un 23,8\%, esto es, mal valorado en un 63,5\%. Las necesidades y limitaciones en infraestructura, así como espacios y materiales didácticos en la educación en prisión es también referida y resaltada por investigaciones previas (Ulate y Vargas, 2012) y consolida otro resultado de esta investigación coherente con el estado de la cuestión.

Aquí también es importante mencionar que los centros penitenciarios son todos muy distintos entre sí y las condiciones pueden ser más o menos deficitarias en cada uno de ellos. Por lo tanto, es imperativo el trabajo en aras de regularizar y estandarizar los espacios de estudio de acuerdo a criterios técnicos y profesionales que potencien el establecimiento de espacios idóneos para la actividad académica.

Tomando en cuenta que el alumnado privado de libertad no puede acceder a la plataforma virtual de la universidad o al internet para acceder a información, conocimientos y recursos didácticos; las malas condiciones de las bibliotecas y de los espacios físicos deben ser prioridad en términos de lo que se debe y puede mejorar. 


\section{Tutorias limitadas o inexistentes}

En relación con las tutorías, la evaluación ejecutado por el estudiantado destaca a un $63,5 \%$ de estos evaluándolas como Muy inadecuadas y un 15,9\% como bastante inadecuadas, esto es un 79,4\% de la muestra otorgando una baja evaluación a las tutorías. Aquí es importante destacar que las tutorías que reciben en prisión son muy limitadas, sin desarrollarse de manera presencial o inexistentes en la mayoría de las materias. Esto es uno de los aspectos más señalado como deficiencia del sistema estudios.

Así, el segundo elemento mayor veces calificado como la principal dificultad es Inadecuadas o inexistentes tutorías con un 63,5\% de valoraciones de 10 . De acuerdo con esto, las y los estudiantes mencionan, al preguntarles por la principal dificultad de su proceso educativo, por ejemplo, "No hay tutorías para evacuar dudas" (Privado de libertad \# 50, comunicación personal, marzo, 2017) o "No se cuenta con profesores para tutorías" (Privado de libertad \# 22, comunicación personal, febrero, 2017). La insistencia en plantear a la ausencia de tutorías como una de las principales dificultades del proceso y por ende como una necesidad inminente es también destacado por Castrillo (2012) y Viedma (2003), entre otros.

\section{Falta de acceso a internet}

La principal dificultad y limitación, de acuerdo con los participantes del estudio, en la educación universitaria en prisiones de Costa Rica, es, la falta de acceso a internet. El acceso a internet es claramente determinado como Muy inadecuado en un 92,1\% de las veces, así como 4,8\% de las veces como Bastante inadecuado. Lo que se explica de acuerdo a la prohibición total que tiene el estudiantado universitario en prisión en Costa Rica para acceder al internet, configurándose como una de las principales demandas y dificultades de las y los estudiantes y una necesidad inminente para la potenciación de sus procesos educativos.

En este sentido, al ser consultado por la principal dificultad de sus estudios en prisión, un 77,2\% de la muestra ha calificado con valoración de 10 a la falta de acceso al internet y que corresponde a la mayor calificación posible para una dificultad, es decir, la principal dificultad en consideración del porcentaje destacado de participantes.

Lo anterior es congruente con lo planteado en multiplicidad de ocasiones por las y los estudiantes en las preguntas abiertas del cuestionario y en la etapa cualitativa de investigación cuando mencionan "El principal problema es no tener acceso a internet, pues muchos trabajos y proyectos deben ser descargados de la plataforma virtual y se debe esperar a que nos envía el material impreso o digital" (Privado de libertad \# 27, comunicación personal, febrero, 2017); o "Para mi es [principal dificultad] el no tener acceso a la plataforma virtual de la UNED, en eso somos estudiantes con una gran desventaja” (Privado de libertad \# 32, comunicación personal, febrero, 2017).

Así, como se presenta en la Figura 7, se determina como principal dificultad la falta de acceso a internet con una media de 8,37; las inadecuadas o inexistentes tutorías con una media de 7,49; biblioteca inexistente o limitada con una media de 6,40; inadecuados o inexistentes materiales didácticos con una media de 5,40 e inadecuados o inexistentes espacios para el estudio con media de 5,30. 


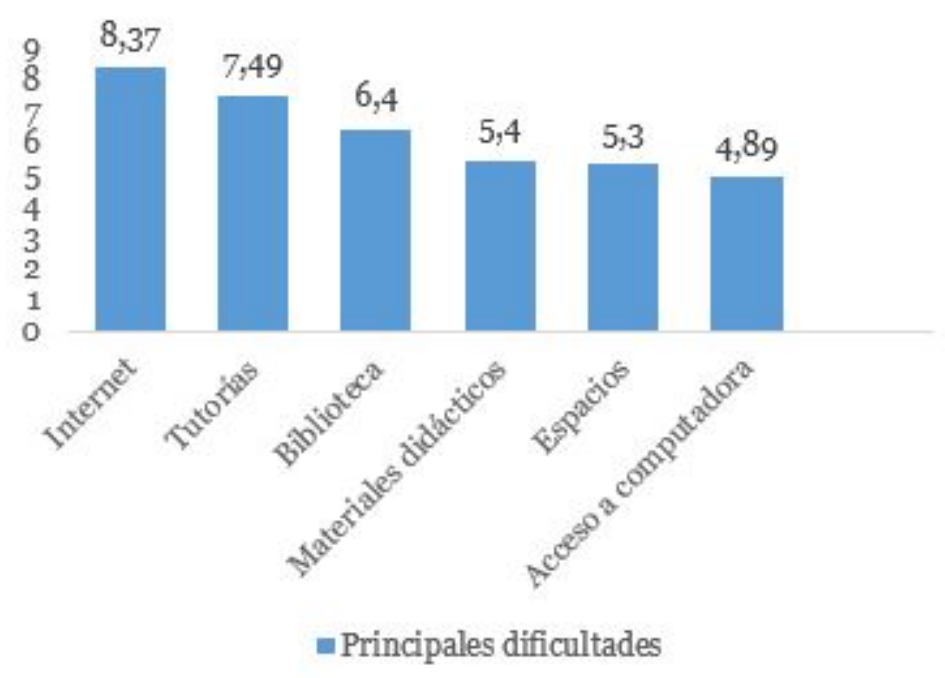

\section{FIGURA 7 \\ Principales dificultades de la experiencia universitaria en prisión \\ Fuente: Elaboración propia}

Con respeto al acceso a internet y las nuevas tecnologías de información es importante reiterar que la imposibilidad de disfrutar de dicha herramienta es una cuestión nacional, una restricción de gobierno y no intrínseca a los procesos educativos en prisión; el acceso a internet con fines académicos es posible en otros lugares del mundo en dónde inclusive avanzan con reflexiones acerca de cómo mejorarlo y que destacan la importancia de dicho acceso (Moreira, Reis-Monteiro y Machado, 2017).

En relación con lo anterior, por ejemplo, destaca lo señalado por Moreira, et ál (2017) cuando menciona que "la combinación de los diferentes métodos y tecnologías de aprendizaje que implican la interacción entre los abordajes educativos y los recursos tecnológicos es esencial para mejorar la calidad de la educación dentro de las prisiones portuguesas" (p.47). En la misma línea, Viedma (2013) y Scarfó y Zapata (2013) hablan de la limitación que significa el inadecuado o inexistente acceso y aprovechamiento de las TIC en los procesos educativos en los centros penales.

Por lo tanto, es posible y necesario, por parte de las instituciones responsables, repensar dicha limitación, evaluar las posibilidades de establecer este acceso de manera coherente con la seguridad institucional y social es posible y requiere la voluntad política de las personas e instituciones responsables. En Costa Rica y en otros países, se podría pensar en plataformas y espacios virtuales de aprendizaje universitario específicos para la población privada de libertad. Al respecto de esto se ha reflexionado con anterioridad en trabajo previo (Murillo, 2017).

Sobre todo, es significativo destacar que las principales dificultades mencionadas se relacionan en tanto plantean a las y los estudiantes dificultades para el acceso a la información. En este sentido, por ejemplo, mencionan "No hay suficiente material para investigar o aclarar dudas" (Privado de libertad \# 50, comunicación personal, marzo, 2017); "Las limitaciones que se tiene en base a tener mejor información para la elaboración de tareas" (Privado de libertad \# 56, comunicación personal, marzo, 2017) o "Siento que mejorar o actualizar las bases de datos para obtener información debido a no poder tener acceso a la internet" (Privado de libertad \# 21, comunicación personal, febrero, 2017).

También es valioso reconocer, como importante dificultad del proceso educativo y relacionada con el acceso a la información, a las relaciones con los funcionarios del centro penal y en especial con los equipos de seguridad. Los participantes relatan las complicaciones que algunas veces se generan a la hora de recibir y 
entregar tareas o documentos académicos y universitarios; así como la dificultad o imposibilidad ocasional para ser trasladados a su aula de estudio cuando el centro penitenciario cuenta con esta. Así, la coordinación al respecto debe ser potenciada y mejorada.

Acoso

Es interesante destacar que un $23,8 \%$ de la muestra de estudio ha mencionado que ha sufrido acoso en el centro penitenciario por ser estudiante. Además, casi idénticos resultados se presentaron al evaluar si ser estudiante en prisión les ha generado algún problema. Esto es 23,8\% menciona que sí y $73 \%$ no.

Si bien, la gran mayoría de la muestra no considera haber sufrido acoso o problemas por su condición de estudiantes, se debe resaltar que un número no menor de personas relatan sí haber sufrido dicha afectación. Estos aspectos requieren futuras profundizaciones para evaluar y diseñar mecanismos para la eliminación de situaciones abusivas y la contención de las personas involucradas.

\section{d) Necesidades}

Con respecto a las principales necesidades que experimentan en el proceso educativo las y los estudiantes universitarios privados de libertad y directamente coherentes con las dificultades anteriormente destacadas, un $92,1 \%$ de la muestra ha calificado el acceso a internet como la principal necesidad en consideración del porcentaje destacado de participantes.

El segundo elemento mayor veces calificado como la principal necesidad es Más y mejores tutorias con un $66,7 \%$ de valoraciones 10 . Visualizado también cuando las y los estudiantes plantean, por ejemplo, La necesidad con respecto a más tutorías y por ende mayor interacción y acompañamiento de tutores concuerda con Sáez, Domínguez y Mendoza (2014), que destacan que:

En una enseñanza a distancia los contenidos y los materiales de aprendizaje son elementos esenciales. El otro elemento o factor clave esencial es la interacción tutor-estudiante. Estas características influyen y determinan la eficacia y el éxito de los programas a distancia. La calidad de la comunicación entre el estudiante y el tutor es crucial para el curso de estudios (p. 199).

De esta forma, en términos de una valoración de la media de los resultados, como se visualiza en la Figura 8, se determina como principales necesidades a la posibilidad de acceder a internet con una media de 9,33; la necesidad de más y mejores tutorías con una media de 8,16 ; la posibilidad de contar con biblioteca o mejorarla con una media de 7,32; más y mejores materiales didácticos con una media de 6,70 y la necesidad de mejores espacios para el estudio con media de 6,54. Así, como expresan, con una media de 5,94, la necesidad de computadora y un acceso idóneo a esta. 


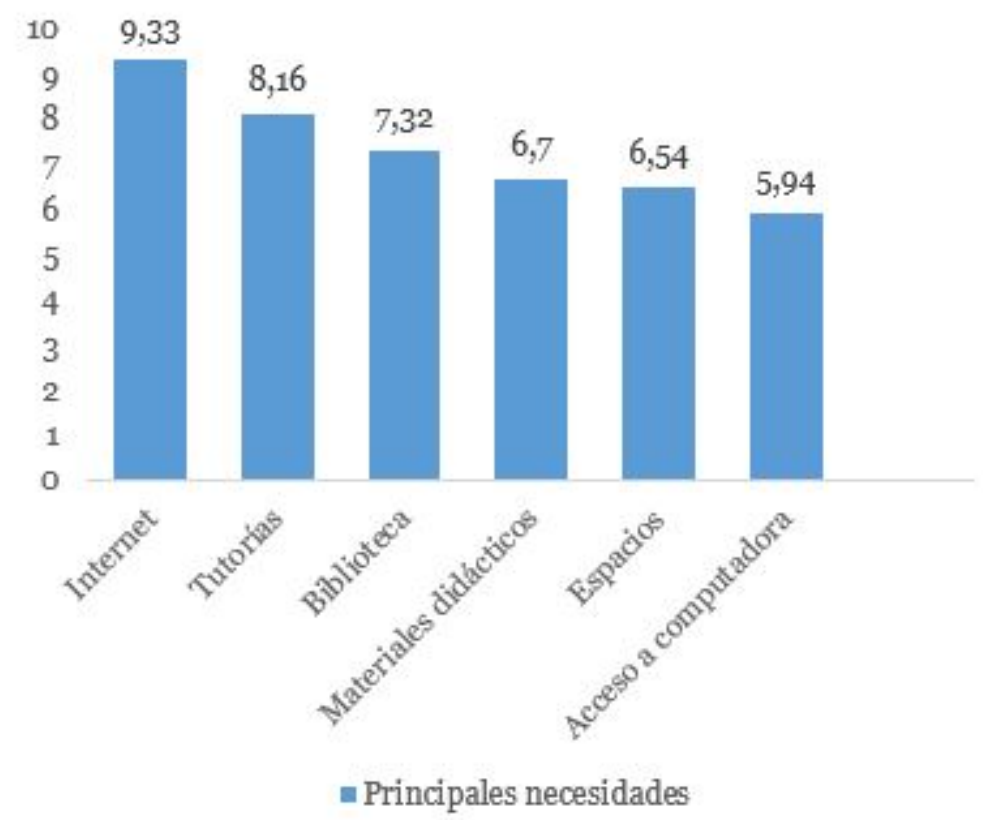

FIGURA 8

Principales necesidades de la experiencia universitaria en prisión

Fuente: Elaboración propia

\section{Conclusiones}

Se puede asegurar que la educación universitaria en prisión es compleja, que a pesar de sus valiosos logros o alcances presenta grandes dificultades y necesidades y, por ende, requiere de una atención específica y especializada para potenciar su desarrollo, mejorar su calidad y asegurar la ejecución del derecho a la educación.

Dentro de los principales logros destaca que la educación universitaria en el contexto penitenciario costarricense se ha mantenido a lo largo de los años y ha logrado consolidar una oferta permanente. Además, se concluye que la educación superior en prisiones de Costa Rica presenta condiciones y procedimientos que la hacen accesible para las y los estudiantes interesados y que cumplan requisitos; accesible sin significativas dificultades y con facilidades para la obtención de beca que cubra los costos de las asignaturas.

Se concluye que la educación en prisión es un factor protector. Por un lado, el aula da sensaciones de mayor seguridad a las y los estudiantes. Además, la educación universitaria en general interviene la cotidianidad penitenciaria, interpela la ociosidad intrínseca a la institución total y previene de los peligros del contexto.

Ahora bien, dentro de las principales dificultades percibidas por el estudiantado destaca la falta de internet, la falta de tutorías y de otras oportunidades para un adecuado acceso a la información. Además, sobresale el escaso acompañamiento académico y limitaciones en términos de infraestructura y espacio físico.

También, es valioso resaltar que, de acuerdo con la perspectiva del alumnado, los funcionarios del centro penitenciario son trascendentales a la hora de potenciar y facilitar el proceso educativo. Tanto los funcionarios de la universidad que trabajan o visitan los centros penitenciarios, así como los funcionarios de las áreas educativas y, muy importante, como se destacó, el personal de seguridad; son elementos claves en el proceso educativo de las y los estudiantes y en potenciar su permanencia y éxito. Los actores/funcionarios antes destacados son muy importantes en tanto facilitan información y acompañan el proceso educativo. 
En lo que respecta a la seguridad, destaca su papel como facilitadores de acceso a documentos, traslado de asignaciones académicas y traslado al aula de la universidad. Es conflictivo y perjudicial que dicho personal de custodia no siempre facilite dichas acciones.

Como principales necesidades de la educación universitaria en prisión resaltan: la obligación modernizar las herramientas didácticas y pedagógicas, así como evaluar y diseñar estrategias para incorporar el internet y las nuevas tecnologías de la información a la dinámica académica en este espacio.

El estudiantado visualiza la necesidad innegable de acceder a mayor información que permita asumir sus asignaciones académicas y su proceso de formación. Así como también resalta la demanda de más acompañamiento en el proceso educativo y por parte de tutores y funcionarios universitarios. Así, es necesario potenciar el acompañamiento a las y los estudiantes, velar porque estos accedan a material didáctico adecuado y actualizado para sus asignaciones académicas, así como estar pendiente de necesidades cotidianas y específicas de las y los estudiantes que son, debido a la dinámica institucional, difíciles de conocer sin un acompañamiento continuo.

Es necesario replantear el proceso educativo y su modelo de trabajo. Discutir acerca de las necesidades en torno al acceso a la información incluyendo el acceso al internet y a las nuevas tecnologías en información. En la educación a distancia el espacio de la plataforma virtual, las bases de datos y los materiales didácticos interactivos y audiovisuales son trascendentales y en esto, la población privada de libertad, enfrenta un gran vacío y una enorme desventaja con respecto a estudiantes fuera de prisión.

La tarea es posible, requiere más investigación, voluntad, decisiones e innovación.

\section{REFERENCIAS}

Arias, D. (1996/1997). Los derechos humanos: un paradigma para la atención en salud de los privados de libertad. Revista latinoamericana de Derecho médico y medicina legal. 1(2), 43-50.

Bandeira, W., Zélia, M., Troccoli, G., Costa de Castro, R., Barbosa, M., J., Gurgel, M. (2008). Proyecto educando para la libertad: la educación en establecimientos penitenciarios bajo el análisis. Educación en Prisiones en Latinoamérica. Brasil: UNESCO. Recuperado de https://www.oei.es/historico/pdfs/prisiones.pdf

Blazich, G., De Milén, S. y Viedma, A. (2007). La educación en establecimientos penitenciarios argentinos: estudio de algunas cárceles de las ciudades de resistencia y corrientes. Revista digital, 1, 1-25. Recuperado de https://b it.ly/2WdNNNa

Camacho, D. (2009). Relaciones Internacionales, Movimientos Sociales y Derechos Humanos. La construcción de una cultura de los derechos humanos en América Latina. Manuscrito inédito. Ciudad de México: México: Acervo Histórico Diplomático, Secretaría de Relaciones Exteriores, México

Camacho, D. (2016). El concepto de derechos humanos. El dilema del carácter de los derechos humanos. Revista de Ciencias Sociales, 2(152), 1-7. Recuperado de http://www.redalyc.org/pdf/153/15348419001.pdf

Castrillo, O. (2012). Aprendizaje de las y los estudiantes Universitarios Privados de Libertad, Centro Penal Calle Real, Liberia. En VIII festival internacional de matemática. Conferencia llevada a cabo en la Universidad Nacional, Sede Chorotega, Costa Rica.

De Castro, B. (2004). Introducción al estudio de los Derechos Humanos. Madrid, España: Universitas S.A.

Goffman, E. (2001). Internados: ensayos sobre la situación social de los enfermos mentales. Buenos Aires: Amorrortu.

Gutiérrez, J., Callejo, J. y Viedma, A. (2010). Estudios superiores en la educación penitenciaria española: un análisis empírico a partir de los actores. Revista de Educación, 353, 443-468. Recuperado de https://bit.ly/2dcv9fV

Instituto Nacional para la Evaluación de la Educación (INEE) (2014). El derecho a una educación de calidad. México: INEE. Recuperado de https://bit.ly/1tMU3rx

López, M. (2009). La educación en las cárceles de la provincia de Buenos Aires. Legislación, violencia y ejercicio. Question 1(27), 1-11. Recuperado de https://bit.ly/2LQJh3y 
Moreira, J., Reis, A., y Machado, A. (2017). La educación superior a distancia y el e learning en las prisiones en Portugal. Revista comunicar 51, 39-49. Recuperado de https://bit.ly/2Jp2fvX

Murillo, W. (2017). Un modelo pedagógico de educación universitaria a distancia para estudiantes en prisión: perspectivas y desafíos. Revista posgrado y sociedad 15(2), 49-64. Recuperado de https://bit.ly/2Wc2Eb4

Organización las Naciones Unidas (2015). Declaración Universal de los Derechos Humanos. Recuperada de https://b it.ly/1TDtaiu

Organización Mundial de las Naciones Unidas (2016). Reglas Minimas de las Naciones Unidas para el Tratamiento de los Reclusos (Reglas Nelson Mandela). Resolución 70/175. Recuperado de https://undocs.org/es/A/RES/70/175

Rivera, M. (2016). Análisis de los factores que inciden en el rendimiento académico de la población estudiantil privada de libertad de los centros de atención institucional Dr. Gerardo Rodríguez Echeverría, La Leticia y Sandoval del diplomado en administración de empresas en el 2015. (Tesis de licenciatura). UNED, Costa Rica.

Sáez, J. M., Domínguez, C. y Mendoza, V. (2014). Valoración de los obstáculos, ventajas y prácticas del e-learning: un estudio de caso en Universidades Iberoamericanas. Educatio Siglo XXI. 32(2). 195-220. Recuperado de http:// revistas.um.es/educatio/article/view/202221

Scarfó, F. (2011). Estándares e indicadores sobre las condiciones de realización del derecho a la educación en las cárceles. (Tesis de maestría) Universidad Nacional de la Plata, Argentina.

Scarfó, F. y Zapata, N. (2013). Obstáculos en la realización del derecho a la educación en cárceles. Una aproximación sobre la realidad argentina. Recuperado de https://bit.ly/2VL515s

Scarfó, F., Cuellar y Mendoza, D. (2016). Debates: sobre el rol de la escuela y de los educadores de adultos en las cárceles. Cad. Cedes, Campinas. 36(98), 99-107. Recuperado de https://bit.ly/2JLFMbT

Ulate, I. y Vargas, E. (2012). La educación a distancia en la profesionalización de las personas con discapacidad y privados de libertad. Revista electrónica Educare. 16(3), 157-173.

Viedma, A. (2003). La educación universitaria en prisión. Estudio de los alumnos de la UNED internos en centros penitenciarios. Revista iberoamericana de educación a distancia 6(2), 97-120. Recuperado de https://bit.ly/2V $\operatorname{LLi} 5 \mathrm{x}$

Viedma, A. (2013). Universitarios en prisión: experiencias y apariencias de sentido en el espacio penitenciario. (Tesis de grado). Universidad Nacional de Educación a Distancia, España.

\section{BY-NC-ND}

